\title{
Enhancing climate resilience of transboundary water allocation agreements: the impact of shortening the agreement's lifetime on cooperation stability
}

\author{
Maryam Jafroudi ${ }^{1}$ (D)
}

Accepted: 27 August 2018 / Published online: 4 September 2018

(c) The Author(s) 2018

\begin{abstract}
Efficient use of transboundary rivers under climate change requires periodic adaptation of their governing regime to the hydrological realities of the basin. Applying a short-term time horizon to an agreement on water allocation can make it easier for the riparian states to update the terms of their cooperation periodically, and thus, adapt them to changes in water availability resulting from climate change. However, agreements with a pre-agreed termination date can only increase the efficiency of water use if they lead to a stable cooperation. In this paper, I analyze how riparian states' previous knowledge of the termination date of a water allocation agreement affects the agreement's stability. The results show that when riparian states are aware of the agreement's termination date, there is a higher chance for them to free-ride. However, certain cases may occur in which previous knowledge of the agreement's termination date can contribute to the stability of an agreement by enforcing compliance.
\end{abstract}

Keywords Climate change - Transboundary rivers - Climate change resilience · International water law agreements $\cdot$ Game theory $\cdot$ Rational decision making

\section{Introduction}

The rise in the number of international agreements on freshwater resources, from 253 in 1963 to more than 400 in 2000 (Wolf 2002), is a testament to how useful states are finding it to regulate their relations with respect to these resources through international agreements. Landmark treaties on transboundary freshwater resources, such as the UNECE Convention on the Protection and Use of Transboundary Watercourses and International Lakes of 1992 and the UN Convention on the Law of the Non-navigational Uses of International Watercourses of 1997, also recognize the role of international agreements in effective transboundary water management, either by requiring states to conclude such treaties

Maryam Jafroudi

mjafrudi@uvt.nl

1 Tilburg, The Netherlands 
(United Nations 1992), or by recommending they consider such a possibility (United Nations 1997).

Concluding international agreements on transboundary freshwater resources has many advantages. These treaties stabilize relations between states with respect to their freshwater systems (McCaffrey 2003). They clarify property rights over water and prevent states from moving toward coercion and grabbing resources from others (Ansink et al. 2015). They pave the way for information exchange. They create potential for the establishment of joint management mechanisms and facilitate the reasonable and equitable use of water. Moreover, basins with institutional mechanisms, like treaties, are less at risk of conflict (Yoffe et al. 2003).

However, there is a downside to managing transboundary freshwater resources through international agreements. While the characteristics of freshwater resources are by nature dynamic and can change drastically, for example, due to changes in rainfall and precipitation patterns, treaties are essentially rigid instruments and can only be modified or terminated under certain limited conditions, for instance under mutual agreement between the parties or when there is a supervening impossibility of performance (United Nations 1969). Thus, the dynamic nature of freshwater resources may clash with the rigidity of international agreements, and drive those states that are negatively affected by the changing conditions toward free-riding.

Climate change is one of the main factors that can alter the characteristics of freshwater systems. It can change the form, intensity and timing of water demand, precipitation and runoff, implying that past climate conditions are no longer a predictor of the future state (Intergovernmental Panel on Climate Change 2014). Therefore, climate change, as a cause of water variability, poses a serious threat to the continuity of cooperation agreements on transboundary freshwater resources.

Aware of these facts, many scholars have searched for possible methods to enhance the flexibility of agreements on transboundary freshwater resources in the face of changing conditions (Cooley et al. 2009; Cooley and Gleick 2011; Dinar et al. 2011; Wilby and Dessai 2010; McCaffrey 2003). The methods suggested have included: Entering into a general "framework" agreement that requires parties to conclude periodic treaties; giving the agreement a short life span; introducing special provisions for droughts and floods; including a provision that allows parties to terminate the agreement upon a given period of notice, using a mechanism akin to the joint contractual plan; and empowering joint institutions to respond to the changed conditions.

This paper focuses on the suggestion of using agreements with short and finite time horizons to enhance the flexibility of water allocation arrangements in the face of climate change. This suggestion has strong theoretical support within the existing literature on agreement flexibility. Bilder (2014) considers short-term and finite agreements to be less of a risk for states. He argues that, as the time horizon increases, the confidence of states in their ability to predict the future decreases. He states that, in the long term, the chance that the circumstances which led to the agreement being reached may cease to exist is perceived to be higher. Furthermore, he argues that the actual and phsychological costs to the states of continued commitment to an agreement that turns out to be disadvantageous may increase in proportion to the length of time that they are held to that agreement. Koremenos (2001) suggests that by limiting the duration of an agreement, states can account for the uncertain contexts in which agreements are made and ideally kept. Harstad (2008) argues that if the future is highly uncertain, a short-term agreement may be more desirable. Additionally, the literature on climate change claims that the changing circumstances of our world make it necessary for states to update their agreements periodically, and that this 
may be easier if an agreement is set to expire, making it natural to negotiate a successor (Harstad 2008).

Against this background, it seems that the use of short-term agreements on freshwater resources increases the efficiency of water management under the impact of climate change by accommodating freshwater resource variability. However, an important question remains unanswered: How will setting an expiry date for agreements on freshwater resources affect the level of compliance by the participating states? The current paper is trying to provide an answer to this question.

An agreement that is not being complied with cannot be an effective instrument to increase the efficiency of water use. Cooperation within the framework of an agreement is stable as long as all parties continue to comply with its provisions. As such, stability of cooperation depends on the incentives for states to comply. Thus, in order to assess the stability of interstate cooperation, the strategic considerations of states during the agreement's implementation phase need to be studied.

This paper uses a game-theoretical model of cooperation to study the incentives of states to comply with their obligations under a water allocation agreement. Such methodology makes it possible to model interstate interactions based on states' strategic concerns and make concise predictions about their future behavior. The focus of the paper is on the compliance of states with their obligations under water allocation agreements in rivers with two riparian states, which compose a large portion of treaties on freshwater resources (Hamner and Wolf 1998). The conclusions reached are only applicable to rivers with two basin states that represent more than 50\% of the rivers worldwide (Dinar 2007). The model of cooperation is based on an upstream-downstream context, relevant to linear rivers shared among states.

The remainder of the paper is structured as follows. The second section is dedicated to presenting the game theoretic baseline model of an agreement on water allocation. The third section extends this naive model to accommodate the complications arising in the implementation phase of the agreement and remedies offered under international law to mitigate them. In the fourth section, the basis of the agreement's stability will be explained by using the extended model. The fifth section looks into the impact that prior knowledge of an agreement's termination date may have on its stability. The conclusion is offered in the seventh section, explaining briefly why shortening the lifetime of the water allocation agreements should not be universally prescribed as a way to increase agreements' resilience to changes in water quantity caused by climate change.

\section{Game theoretic baseline model}

Assume a river is shared by two states $i \in\{u, d\}$. The river has its origin in the upstream state $u$ and flows through the downstream state $d$. The expected streamflow of the river that can be allocated between the states is denoted by $e_{u}$.

Each state $i$ expects to use $x_{i}, 0 \leq x_{i} \leq e_{u}$ and $x_{u}+x_{d} \leq e_{u}$ amount of water, leading to a certain amount of net benefit. This benefit is determined by a function $b_{i}\left(x_{i}\right): \mathbb{R}_{+} \rightarrow \mathbb{R}$. I assume that this function is increasing, differentiable for each $x_{i}>0$ and strictly concave. This suggests that $b_{i}^{\prime \prime}\left(x_{i}\right)<0$. The implications of such assumptions regarding the benefit function are that more water input results in higher benefits for the states. However, the increase in benefits generated by each unit of water input 
does not increase as more input is used. For instance, if water input increases from 5 to 6 units, benefits may increase by 2 units. But, if water input increases from 9 to 10 units, benefit increases by less than 2 units.

As states are individually rational, they tend to maximize their benefits from water use. However, their ability to do so is subject to the limitations imposed by the water availability. Since the streamflow is unidirectional, the ability of $u$ to maximize its benefit is completely dependent on $e_{u}$. Given that $u$ has the first option to use the river, the availability of water to $d$ is constrained by $u$ 's consumption of the streamflow. State $d$ can try to increase its water availability and approach benefit maximization by persuading the upstream state to use less water.

I assume that, given the amount of $u$ 's expected usage of $e_{u}$, the forecasted streamflow left for $d$ does not suffice for maximization of $d$ 's benefit function, leading $d$ with a desire to negotiate a water allocation agreement with $u$. Concluding a water allocation agreement entails $d$ 's access to an extra amount of water, boosting its expected benefit.

If I denote the benefit that each state expects from the use of a share of $e_{u}$ with $B_{i}=b_{i}\left(x_{i}\right)$ and use the superscripts $c$ and $n$ to represent cooperation and noncooperation, respectively, then entering into a treaty on water allocation for $d$ means:

$$
B_{d}^{\mathrm{c}}>B_{d}^{\mathrm{n}}
$$

Meanwhile, letting $d$ receives a larger share of $e_{u}$ constrains water availability to $u$. Therefore, if $u$ agrees to a water allocation scheme it follows that $u$ shall restrict its maximization behavior. In terms of net benefit function this means that:

$$
B_{u}^{\mathrm{c}}<B_{u}^{\mathrm{n}}
$$

To make the cooperation attractive to the upstream state, the downstream state $d$ shall compensate for the foregone benefits of $u$. Compensation measures, monetary or otherwise, also referred to as non-water transfers, are commonly used in water-related agreements such as the agreement between the Republic of the Sudan and the United Arab Republic for the full utilization of the Nile waters. Offering compensation does not contradict the principle of limited sovereignty of states over shared freshwater resources, which requires the upstream state to let some water pass to the downstream state; rather it is used as an incentive for joining the agreement and encourages compliance (Finus 2003).

Given these considerations, an agreement on water allocation between the two states shall define the share of water for each state from the allocable flow, and the value of the non-water transfer that the downstream state "pays" to the upstream state as an incentive for cooperation. Following Ansink and Ruijs (2008), I assume that the value of non-water transfer, denoted by $\breve{v}_{u}^{c}$, is equal to the value of compensation of $u$ for foregone benefits and a share of $\Phi \in[0,1]$ of the additional benefits from cooperation:

$$
\breve{v}_{u}^{\mathrm{c}}=\Phi_{u}\left[\left(b_{d}^{\mathrm{c}}\left(\check{x}_{d}^{\mathrm{c}}\right)-B_{d}^{\mathrm{n}}\right)+\left(b_{u}^{\mathrm{c}}\left(\check{x}_{u}^{\mathrm{c}}\right)-B_{u}^{\mathrm{n}}\right)\right]+\left(B_{u}^{\mathrm{n}}-b_{u}^{\mathrm{c}}\left(\check{x}_{u}^{\mathrm{c}}\right)\right)
$$

Note that in this equation $\check{x}_{i}^{c}$ stands for the amount of water allocated to state $i$ under cooperation. This method of calculating non-water transfers is related to the asymmetric Nash bargaining solution (Binmore et al. 1986) and determines the relationship between the values of $\check{x}_{d}^{\mathrm{c}}, \check{x}_{u}^{\mathrm{c}}$ and $\breve{v}_{u}^{\mathrm{c}}$, such that it fulfills the requirement of Pareto efficiency. This means that as long as the values of $\breve{x}_{d}^{\mathrm{c}}, \breve{x}_{u}^{\mathrm{c}}$ and $\breve{v}_{u}^{\mathrm{c}}$ are determined in order to satisfy Eq. (3) and no exogenous changes are made to the system, none of the states can be made better off under any other agreement over the amount of water and non-water transfer without the other state becoming worse off. An agreement which satisfies (3) is stable by design. For the rest of this paper, I assume that $u$ and $d$ have agreed to allocation terms satisfying (3). 


\section{Challenges to cooperation stability and remedies under international law}

Compliance with the terms of a treaty does not automatically follow from ratification by the states which are party to it. Empirical evidence includes a relatively large body of ratified treaties that were not complied with afterward. The list of examples in the field of environmental law includes some famous treaties such as the Kyoto Protocol (Barrett 2003; Barrett and Stavins 2003), the Helsinki Protocol on Reduction of Sulfur of 1985, the Convention on International Trade of Endangered Species (McEvoy and Stranlund 2006; Sand 1997), the International Convention for Regulation of Whaling (Heister 1997) and the Oslo Protocol on Sulfur Reduction (Barrett 2003). This shows that, while consent is a necessary condition for the states to be bound, it is not sufficient.

There are different reasons for states' noncompliance with environmental agreements, including lack of capacity and resources, lack of diligence and lack of will (Rinceanu 2000). The latter factor is the most frequent cause of deviation (Hovi and Areklett 2004) and has a greater importance as it impedes the implementation of due diligence even if the requirements of sufficient capacity and resources are met.

Consent to an agreement and the will to enforce it are two separate concepts. Consent is produced during the negotiation phase as the result of a belief that, given everyone complies with its obligations, the welfare of all parties will improve. In the model proposed in this work, consent is produced since, if both $u$ and $d$ comply with their obligations, $d$ will be able to improve its welfare by receiving an extra amount of water, while $u$ will obtain higher welfare by receiving non-water compensation in exchange. Nevertheless, while an agreement may be worthy of initial consent, states may find themselves better off freeriding, either on the other state's virtuous behavior (Neumayer 2017; Barrett 1995) or due to nonconformity between expectations and reality.

In the agreement presented herein, states will receive higher payoffs if they both comply. However, both $u$ and $d$ will benefit more from free-riding if the other fulfills its obligations. In case of $d$ 's obedience and $u$ 's noncompliance, $u$ will gain the expected benefit of noncooperative use of water plus the non-water transfer made by $d$. Meanwhile, if $u$ complies and $d$ deviates, $d$ will obtain the benefits of the extra water it receives from the upstream without incurring any cost.

Even without such free-riding incentives, the robustness of cooperation under the agreement may be threatened in the implementation phase due to discrepancies between expectations and reality. The water allocation agreement, as explained, is designed based on the expected amount of river streamflow. The expected amount of allocable flow may, however, not be the same as the actual flow observed in the implementation phase, which may eventually affect the payoff of the parties and cause them to consider deviation as the better option.

It is therefore important to see what remedies are available to mitigate these free-riding incentives in the implementation phase. A solution to the problem of noncompliance should deal with the origin of the temptation to free-ride. Incentives for free-riding are the result of the perception that the benefits of deviation surpass those of compliance. However, if a state is convinced that deviation will lead to a less favorable payoff, it may not be inclined to commit a transgression.

On the domestic level, governments use coercive measures such as police forces, fines, jail and so on to convince their subjects that deviation is less beneficial than compliance. Such coercive measures are not readily available at the international level. International 
organizations have "no jail house, no bail bondsmen, no blue helmets, no truncheons or tear gas" (Bello 1996). The Security Council may impose some costs on a deviating state by adopting sanctions or prescribing the use of force under Chapter VII of the UN Charter. Nonetheless, such intervention from the Security Council occurs only in the face of a serious threat to world peace and security. However, it is wrong to assume that violating international law is not associated with any costs.

A free-rider may face some costs arising from loss of reputation. ${ }^{1}$ Within the international community, states are not living in isolation; their survival is dependent on their relationships with their counterparts, which can be damaged by exposing their unreliability through defection from the terms of the agreement (Guzman 2008; Goldsmith and Posner 1999, 2005; Hathaway 2005).

Additionally, under international law, countermeasures can be used to create some cost against a free-rider. International law authorizes an injured state to perform an act that would otherwise be contrary to its international obligations vis-a-vis the deviator in order to produce compliance and procure reparation (International Law Commission 2001).

Violation of international law can also incur costs as a result of an unfavorable decision by an international court or tribunal. States can either agree to refer future disputes to such institutions, or arrange for such a referral after a dispute is raised. Under either circumstance, the decision of the dispute settlement authority is binding for all parties involved.

More recent environmental agreements have turned to a relatively new cost-creating mechanism that includes penalties for noncompliance, via inclusion of guidelines on how to sanction a deviating state within the treaty itself. The Montreal Protocol on depletion of the ozone layer is the best-known example of such an approach (McEvoy and Stranlund 2006). Sanctions prescribed under such agreements can be carried out by the parties themselves or by the institutions erected, accepted and empowered by them. ${ }^{2}$ However, treatyprescribed sanctions are still uncommon as they are seen to be flawed in that, under most agreements, the deviating state can withdraw from the agreement before the sanction is targeted (Finus 2003). It is worth mentioning that water agreements rarely contain explicit penalties for violations (Stinnett and Tir 2009).

Consequently, the most effective mechanisms available to prevent states deviating from transboundary water agreements and stabilize cooperation within the agreement's framework remain the classic deterrents of reputation loss, countermeasures and dispute settlement mechanisms. The next section explains the implications of these mechanisms for states' decision making during the implementation phase.

\section{Analysis of the baseline model under complications arising during the implementation phase}

As has been argued, in the implementation phase, the decision of states to either comply or deviate is formed by their evaluation of the payoff they will receive under each possible scenario, given the facts on the ground. A state will comply if, independent of what

\footnotetext{
1 Reputation is also referred to as the "shadow of the future." This concept is different from the concept of "global standing" or "popular reputation" of a state. Brewster (2009) offers a comprehensive study of these differences.

2 Kyoto Protocol under Marrakesh Accords provided for the creation of an independent Compliance Committee with the power of applying sanctions in cases of noncompliance.
} 
Table 1 The rows represent actions taken by $u$ and the columns represent actions taken by $d$

\begin{tabular}{lll}
\hline & $\mathrm{c}$ & $\mathrm{n}$ \\
\hline $\mathrm{c}$ & $B_{u}^{\mathrm{c}}+\check{v}_{u}^{\mathrm{c}}, B_{d}^{\mathrm{c}}-\breve{v}_{u}^{\mathrm{c}}$ & $B_{u}^{c}, B_{d}^{c}$ \\
$\mathrm{n}$ & $B_{u}^{\mathrm{n}}+\check{v}_{u}^{\mathrm{c}}, B_{d}^{\mathrm{n}}-\breve{v}_{u}^{\mathrm{c}}$ & $B_{u}^{\mathrm{n}}, B_{d}^{\mathrm{n}}$ \\
\hline
\end{tabular}

the counterpart does, its payoff for compliance is evaluated to be higher than its payoff for deviation.

In general, and based on the model set out in this paper, three different factors may affect the stage payoff of a state during the implementation phase, under different scenarios of compliance and noncompliance:

(1) Benefits the state receives from the use of the observed amount of water;

(2) Costs and benefits arising from non-water transfer;

(3) The consequences of noncompliance expected by the state.

The two first factors on this list determine the direct benefits for states offered by the agreement under different scenarios, which can be seen from Table 1.

The remaining factor influencing the state's decision on compliance is the value of the consequences of deviation. This value is typically difficult to determine, because it inevitably depends upon the actions, capabilities and motivation of the non-deviating states. Furthermore, noncompliance may go undetected. Detected deviation may go unpunished, and even when the cost of uncooperative behavior is certain, its magnitude is dependent on many factors that the deviator may not have full knowledge of. Therefore, to determine the value of the consequences of noncompliance, the deviator should form a belief on whether and how its counterparts would "punish" it due to the deviation. Such a belief has to deal with the uncertainties arising from:

(1) Probability that a counterpart detects the deviation;

(2) Probability that a counterpart imposes a sanction against the free-rider, should the deviation be detected;

(3) Severity of the sanction imposed by the counterpart if the noncompliance is detected.

States can depend on the information they have on their counterpart, including the counterpart's history of interactions with different states, its history of bringing disputes to court or arbitration, its economic power, and its history of reactions to past deviations as an indication of how to assess each of these probabilities.

At each stage $t$, state $i$ begins to estimate the cost it would have to face if it did not comply with the agreement by associating a value to the probability that its noncompliance is detected by the counterpart. State $i$ is aware that it has to pay a price for noncompliance if, and only if: First, the deviation is detected; and second, the counterpart imposes a sanction after detecting the deviation. Thereby, depending on the information available to it at stage $t$, state $i$ associates a probability $p_{i t}(\Delta \cap \alpha), p_{i t}(\Delta \cap \beta)$ and $p_{i t}(\Delta \cap \gamma)$ with the costs arising from incurring sanctions through loss of reputation, $\alpha$, countermeasures, $\beta$ and dispute settlement mechanisms, $\gamma$, respectively. (Note that here $\Delta$ stands for detecting the deviation.)

By definition:

$$
p_{i t}(\Delta \cap \alpha)=p_{i t}(\Delta) p_{i t}(\alpha \mid \Delta), \quad p_{i t}(\Delta \cap \beta)=p_{i t}(\Delta) p_{i t}(\beta \mid \Delta), \quad p_{i t}(\Delta \cap \gamma)=p_{i t}(\Delta) p_{i t}(\gamma \mid \Delta)
$$


Table 2 Stage payoffs of states, the rows represent actions taken by $u$ and the columns represent action taken by $d$

\begin{tabular}{lll}
\hline & $\mathrm{C}$ & $\mathrm{N}$ \\
\hline $\mathrm{C}$ & $B_{u t}^{\mathrm{c}}+\check{v}^{\mathrm{c}}, B_{d t}^{\mathrm{c}}-\breve{v}^{\mathrm{c}}$ & $B_{u t}^{\mathrm{c}}, B_{d t}^{\mathrm{c}}-E\left(k_{d t}\right)$ \\
$\mathrm{N}$ & $B_{u t}^{\mathrm{n}}+\check{v}^{\mathrm{c}}-E\left(k_{u t}\right), B_{d t}^{\mathrm{n}}-\check{v}^{\mathrm{c}}$ & $B_{u t}^{\mathrm{n}}-E\left(k_{u t}\right), B_{d t}^{\mathrm{n}}-E\left(k_{d t}\right)$ \\
\hline
\end{tabular}

Finally, state $i$ associates a magnitude, $k_{i t}^{r}, r \in\{\alpha, \beta, \gamma\}$, to each type of sanction imposed against it. Eventually, the total cost that a state expects it will have to pay for deviation will be calculated as follows:

$$
E\left(k_{i t}\right)=p_{i t}(\Delta)\left(p_{i t}(\alpha \mid \Delta) k_{i t}^{\alpha}+p_{i t}(\beta \mid \Delta) k_{i t}^{\beta}+p_{i t}(\gamma \mid \Delta) k_{i t}^{\gamma}\right)
$$

Accordingly, the stage payoff of states at time $t$ can then be represented as Table 2 .

\section{Basis of agreement stability during the implementation phase}

The agreement between $u$ and $d$ requires them to repeatedly comply with certain obligations for a period of time culminating at $T .^{3}$ This means that at each decision point, $t \leq T$ the two states have to decide between compliance and deviation. The cooperation will be stable if and only if at every decision point both states opt for compliance. This will happen as long as the action set $(c, c)$ represents the unique Nash equilibrium of Table 2.

Proposition Let the following conditions be fulfilled. Then $(c, c)$ will be the unique Nash equilibrium of Table 2.

Condition 1: $B_{u t}^{\mathrm{n}}-B_{u t}^{\mathrm{c}}<E\left(k_{u t}\right)$

This means that, no matter what action is taken by $\mathrm{d}$, the costs expected by $\mathrm{u}$ for noncompliance at $\mathrm{t}$ shall be higher than the benefits it will gain if it deviates.

Condition 2: $\breve{v}^{\mathrm{c}}<E\left(k_{d t}\right)$

This means that at $t$, state $d$ shall expect to pay a cost higher than the "price" it will have to pay under the agreement if it deviates.

Proof I first prove that $(c, c)$ is a Nash equilibrium. This is apparent from Table 2. Given the payoffs, if conditions (1) and (2) hold then none of the states will have an incentive to switch its strategy from compliance to deviation.

In order to prove that $(c, c)$ is the unique Nash equilibrium of the game, I use proof by contradiction. Let $(n, n)$ be a second Nash equilibrium of the game. This creates the following assumption:

$$
B_{u t}^{\mathrm{n}}-B_{u t}^{\mathrm{c}}>E\left(k_{u t}\right)
$$

and

\footnotetext{
${ }^{3}$ Note that unlike the common assumption within game theory literature, in real-world renegotiation of the terms of an agreement is not possible without mutual consent of the state parties. Given the setting of the model for this paper, mutual consent to renegotiate the agreement terms may not be achieved since any agreement that makes one party better off would lead to the other party being worse off.
} 


$$
\check{v}^{\mathrm{c}}<E\left(k_{d t}\right)
$$

Both Eqs. (6) and (7) go against Conditions 1 and 2. Therefore, $(c, c)$ and $(n, n)$ cannot be simultaneous Nash equilibria of the game.

Similarly, if $(c, n)$ is the additional Nash equilibrium, then:

$$
B_{u t}^{\mathrm{n}}-B_{u t}^{\mathrm{c}}<E\left(k_{u t}\right)
$$

and

$$
\breve{v}^{\mathrm{c}}>E\left(k_{d t}\right)
$$

Equation (9) goes against Condition 2. Therefore, $(c, c)$ and $(c, n)$ cannot be simultaneous Nash equilibria of the game. The same line of reasoning shows that $(n, c)$ is not a Nash equilibrium, because it suggests:

$$
B_{u t}^{\mathrm{n}}-B_{u t}^{\mathrm{c}}>E\left(k_{u t}\right)
$$

and

$$
\check{v}^{\mathrm{c}}<E\left(k_{d t}\right)
$$

As can be seen, Eq. (10) goes against Condition 1.

Therefore, it can be concluded that cooperation under the water allocation agreement is stable at a stage if and only if:

$$
\forall t, t \leq T, B_{u t}^{\mathrm{n}}-B_{u t}^{\mathrm{c}}<E\left(k_{u t}\right) \text { and } \check{v}^{\mathrm{c}}<E\left(k_{d t}\right)
$$

\section{Impacts of knowledge of the agreement's termination date on stability}

Following Eq. (12), the subsequent factors can be identified as the elements that shape the stability of cooperation during the period $t=0$ to $t=T$ :

(1) The difference between the benefit function of compliance and noncompliance for the upstream state;

(2) The value of non-water transfer;

(3) The expected cost of noncooperation.

If knowledge of the agreement's termination date affects the value of any of these factors, it could potentially impact the stability of cooperation under the agreement. 


\subsection{Impacts of knowledge of the agreement's termination date on the difference between the benefit function of compliance and noncompliance for the upstream state}

The benefit that the upstream state obtains from the use of water at each hydrological period is the result of the upstream state's ability to put the water it receives into use. Therefore, such a benefit is independent of the timeframe of any water allocation agreement. Thus, previous knowledge of water allocation agreements has no impact on the value of such a benefit. Therefore, the parties' knowledge of the termination date of an agreement would not impact the stability of their cooperation by impacting the difference between the benefit function of compliance and noncompliance for the upstream state.

\subsection{Impacts of knowledge of the agreement's termination date on the value of non-water transfer}

The value of non-water transfer under the present model is defined by Eq. (3). The components of this equations are the benefit functions of upstream and downstream states $B_{u}^{\mathrm{c}}, B_{d}^{\mathrm{c}}, B_{u}^{\mathrm{n}}$ and $B_{d}^{\mathrm{n}}$, as well as the upstream state's bargaining power, $\Phi_{u}$. As such, each of these components are exogenously given from the timeframe of an agreement and, thereby, knowledge of the agreement's termination date will not affect their value.

\subsection{Impacts of knowledge of the agreement's termination date on the expected cost of noncompliance}

Following Eq. (5), each state's expected cost for noncompliance is affected by: First, the probability that it is sanctioned through the available mechanisms of reputation, dispute settlement mechanisms and countermeasures should its deviation be detected; and second, the impact of a possible sanction. Furthermore, following Eq. (4), the probability that a sanction is imposed against a state is dependent first and foremost on whether the deviation is detected.

\subsubsection{Detecting a deviation}

Detection of a deviation is in essence a task. To form a belief over the possibility of the counterpart's detection of $i$ 's deviation, state $i$ has to assess how likely it is for the counterpart to perform such a task. According to Eskins (2012), for any agent to perform a task, it has to have the required opportunity, willingness and capability. Opportunity includes the existence of all necessary variables that make the performance of the task possible; willingness reflects the counterpart's desire to perform the task; and capability captures the counterpart's ability to perform the task given the willingness and opportunity.

In detecting a deviation, having opportunity means having access to data that shows whether the counterpart has complied with its obligations under the agreement. Under the model presented in this paper, the opportunity to detect deviation is always present. The upstream state can always check whether it has received the non-water transfer it is entitled to and the downstream can always check the amount of water that has passed to it from the counterpart. Furthermore, the existence of such opportunity for the states is independent 
of the timeframe of the agreement. Even after the agreement is terminated, states can still check the amount of water and non-water that they have received for the last period when the agreement was in place. Thereby, knowledge of the agreement's termination date does not affect the opportunity to detect the deviation.

However, the case for willingness to detect can be deadline-sensitive. Since states are rational agents, their willingness to perform a task is formed by the utility of the task performance, the value of which is determined by the costs and benefits that the task entails. In the case presented in this paper, the cost of detecting a deviation depends on the facts on the ground (e.g., the cost of utilizing the technology put in place for measuring the amount of water passed from the upstream state) when the task is being performed. Consequently, although the costs of detecting a deviation may vary from period to period, knowledge of a deadline would have no impact on them.

Nevertheless, the benefits of detecting a deviation depend on the course of action that states may consider taking in future if a deviation is detected. For instance, state $i$ may wish to consider imposing a countermeasure or putting forward a legal dispute against its counterpart, should the counterpart deviate from its obligations under the agreement. The existence of a pre-known termination date for an agreement may limit the set of possible future actions that state $i$ can take, in particular in the last implementation phase, after which states are no longer legally entitled to use the option of countermeasures. Thus, states' ability to detect noncompliance and their willingness to conduct a deviation detection activity may decrease due to the knowledge of the agreement's termination date. This potential decrease may be reflected in the states' assessment of $p_{i t}(\Delta)$, for instance if the course of action available to state $i$ in the face of noncompliance is limited to imposing countermeasures against the deviator.

Meanwhile, and similar to the consideration of a state's opportunity, a state's capability to detect violations depends on the facts on the ground and is independent of the agreement's timeframe, although it may vary from period to period. For the downstream state, the capability to detect violations means having access to the technology and knowledge required to compare the amount of water it receives from the upstream state with the amount of water it is entitled to under the agreement. For the upstream state, on the other hand, the existence of a state's capability to detect a violation depends on whether it has the mechanisms at its disposal through which it can assess the value of non-water transfer it has received and compare it to the amount it should have received under the agreement.

\subsubsection{Imposing a sanction}

The opportunity-willingness-capability ontology also helps to identify the impacts of previous knowledge of the agreement's termination date on the probability of sanctions being imposed. Again, the imposition of sanctions against a deviator is a task; consequently, the possibility that state $i$ 's counterpart will perform such a task if a deviation is detected depends first of all on its opportunity to impose such sanctions.

The opportunity to impose sanctions through revising a deviator's reputation depends on the existence of interstate relations or perspective of future relations. Consequently, as interstate interactions go beyond a single agreement, the opportunity to punish a deviator through revising its reputation is likely to remain when the agreement hits its termination date. Therefore, it is unlikely that knowledge of an agreement's termination date impacts the opportunity of the parties to impose a sanction against their counterpart, should it fail to comply with its obligations. 
In the case of sanctions through the use of dispute settlement mechanisms, the existence of opportunity results from the presence of a competent dispute settlement authority and the consent of the states to defer to such authority if a dispute arises. As it is customary under international agreements, clauses that permit referral to dispute settlement authorities survive the termination of an agreement and therefore are not affected by the termination or knowledge of the termination date.

Nevertheless, the existence of an opportunity to use countermeasures to sanction a deviator is of particular interest because of the role that the timing of such measures plays in defining their legitimacy. Under international law, countermeasures are only legitimate when they are used as a means to halt the deviation and not as a punitive recourse. Therefore, the opportunity to sanction a deviator through countermeasures ceases to exist at the final round of an agreement. Consequently, states' knowledge of the agreement's termination date would mean they considered it impossible that their deviation would incur a countermeasure should it be detected. In other words, if states know when $T$ is, they are assured that $p_{i T}(\beta \mid \Delta)=0$. Nevertheless, if there is no knowledge of the final round of the agreement the states may still consider their counterpart as having an opportunity to respond to their deviation via impositions of countermeasures. This means that if $T$ is unknown, $p_{i T}(\beta \mid \Delta) \geq 0$.

Meanwhile, a state's desire to impose a sanction against a deviator depends on the costs and benefits of the sanction imposition. Benefits of sanction imposition are crystallized through achievement of sanction goals. Sanctions can recover the losses suffered by the sanctioning state, they can cause the cessation of the free-riding behavior, or they can do both. However, when the termination date of an agreement is previously known to the parties, sanctions can also benefit the sanctioning state by shaping its future relations with the deviator. A state may decide to impose severe sanctions following a deviation by its counterpart in order to secure a better deal with the free-rider beyond the termination of the agreement. If the agreement has no clear end date, imposing sanctions cannot have such strong "manipulative" power.

A similar statement can be made about the cost of imposing sanctions against others, which may entail some direct or indirect costs for the sanctioning party. The sanctioning state may have to bear some costs in order to refer a dispute to a competent authority, or it may deprive itself of the benefits of an agreement it has put on hold as a response to the deviation it has suffered. Meanwhile, if the end of the agreement is previously known, an additional cost of imposing sanctions can arise out of depriving the sanctioning state of future relations with the deviator. If such a cost is high enough, the state which has suffered the deviation may be reluctant to react harshly to noncompliance, thus securing itself a future relationship with the free-rider.

Consequently, previous knowledge of the termination date of an agreement has the power to shift the willingness of states to impose sanctions both in a positive and a negative direction. This inevitably will be reflected in $i$ 's estimation of $p_{i T}(\alpha \mid \Delta), p_{i T}(\beta \mid \Delta)$ and $p_{i T}(\gamma \mid \Delta)$.

The capability of states to impose sanctions, however, is independent of time constraints. This ability ${ }^{4}$ arises from states sovereignty, which is clearly exogenous to the agreement's termination date.

\footnotetext{
4 The ability of states to impose sanctions shall not be confused with their financial capacity to afford the imposition of sanctions. The latter is captured through the states' willingness to do so.
} 


\subsubsection{Magnitude of sanctions}

When a state decides to impose a sanction against a deviator, it should take into account a series of factors before deciding on the severity of the sanction. In order to work effectively, sanctions should be designed to be harsh enough in order to achieve their goal, by either causing the cessation of agreement violation or recovering the losses. International law requires sanctions to be proportionate to the harms caused. Moreover, state rationality imposes further restrictions on the magnitude of sanctions by requiring states to balance the cost and benefits of the sanction regime in their favor.

For sanctions that are imposed through dispute settlement mechanisms, the magnitude is always decided by a third party. Therefore, the states' knowledge of the termination date of an agreement will not affect its value. This is not the case for the remaining types of sanctions.

When responding to the deviation of a counterpart through reputational sanctions and countermeasures, one factor playing a major role in a state's decision regarding the severity of the sanction is the utility function that each of these types of sanctions has. This utility function depends on the costs and benefits of the magnitude of a sanction, and therefore might be affected by a knowledge of the agreement's termination date.

In the context of a finite agreement, choosing more severe sanctions may cost the sanctioning state a future (more favorable) relation with the targeted state. This cost is something that is not ordinarily expected in the context of an agreement whose termination date is unknown. On the other hand, more severe sanctions in the context of a finite agreement may have additional benefits for the sanctioning state that may not be the case with an agreement with a virtually infinite time-scale. The severity of the sanction can be used as a strategy to intimidate the targeted state into accepting future (and more beneficial) deals with the sanctioning state.

The implications of such a statement for the values of $k_{i t}^{\alpha}$ and $k_{i t}^{\beta}$ are as follows: if based on the available information state $i$ believes that its counterpart may use intimidation techniques for securing a better deal in future should the opportunity arise due to $i$ 's deviation, it will associate a higher value to $k_{i t}^{\alpha}$ and $k_{i t}^{\beta}$ within the context of a finite agreement. ${ }^{5}$ On the contrary, if $i$ feels the counterpart will permit deviation in light of new (better) agreements in future, the value of $k_{i t}^{\alpha}$ and $k_{i t}^{\beta}$ within the context of an agreement with a pre-known termination date will be lower than their respective value within the context of an infinite one.

\subsubsection{Implications for agreement's stability of knowing the termination date}

The impact of states' knowledge of the agreement's termination date on the probability of detecting a deviation, the probability of sanctioning a detected deviation and the magnitude of sanctions result in one general conclusion: knowledge of the agreement's termination date may have serious implications for the value of $E\left(k_{i t}\right)$.

Such knowledge can improve the stability of cooperation within the context of a finite agreement, where both states see each other as capable and willing to use intimidation as a way to secure (better) future deals. Following the conclusions based on opportunity-willingness-capability ontology, with finite agreements the probabilities of detecting deviation, facing reputational sanctions or sanctions through dispute settlement mechanisms, as

\footnotetext{
${ }^{5}$ As long as $k_{i t}^{\alpha}$ stays proportionate to the harm caused by the act of deviation.
} 
well as the magnitude of sanctions through reputation loss and countermeasures, may be perceived as higher than the probabilities and magnitude of the same when the agreement is infinite. Consequently, $E_{\mathrm{fin}}\left(k_{i t}\right)>E_{\text {inf }}\left(k_{i t}\right)$. Thus, there is a higher chance of Conditions (1) and (2) being met when the agreement has a known termination date.

The knowledge of the agreement's termination date may also work against its stability. This is the case when at least one of the states is perceived by the counterpart as reluctant to use sanctions, so as not to lose the chance of a (better) future deal. In such cases, probabilities of deviation detection, $p_{i t}(\Delta)$, facing a reputational sanction, or a sanction through dispute settlement mechanisms should the deviation be detected, i.e., $p(\alpha \mid \Delta), p_{i t}^{\gamma}$, as well as the magnitude of sanctions through reputation, $k_{i t}^{\beta}$, and countermeasure, $k_{i t}^{\alpha}$, will decrease, leading for a reduced chance of at least one of the Conditions (1) and (2) holding true.

A similar pattern of compliance within the context of finite and infinite agreements is also conceivable when both of the states are convinced that the other has no intention of influencing the course of negotiations over future agreements by responding in either a more permissive or severe way to the deviation.

However, even if both states believe their counterparts will use intimidation techniques to secure a better agreement in future, or when a similar pattern of compliance is expected during $t<T$, the finite agreement may still be more prone to instability at its final phase $T$, after which the state suffering from the deviation has no chance of recourse to countermeasures against the free-rider.

Therefore, in general, more scenarios are imaginable where the inclusion of an expiration date in an agreement on water allocation leads to less stability in interstate cooperation.

\section{Conclusion}

International agreements on water allocation are often rigid instruments with nonflexible provisions. The rigidity of these instruments often comes into conflict with the dynamic nature of freshwater resources, especially given that these agreements are only renegotiable under limited and special circumstances. Climate change, in particular, causes severe changes in annual and monthly flow regimes and low flow extremes, threatening the stability of state cooperation under nonflexible water allocation schemes.

The literature on flexibility of water treaties proposes to make these agreements adaptable to changes that can occur over time, by inserting a provision that stipulates a certain termination date, after which the states involved may redefine their water rights according to the basin's new hydrological realities. Ideally, this termination date is defined such that during the lifecycle of the agreement, hydrological changes in water supply remain negligible, so no real need for renegotiation during the lifetime of the treaty arises. Such a proposal paves the way for improving the efficiency of water allocation agreements in the face of climate change. However, to the knowledge of the author, no research has been carried out to assess whether and how setting an expiration date for international agreements on water allocation will affect compliance.

The literature on game theory shows that the strategies of rational decision-makers, such as states, may change depending on whether the duration of their action is known or not. For instance, in a typical finite Prisoner's Dilemma, the dominant strategy of the players at each stage is to defect. However, if the game is repeated infinitely, or if agents remain uncertain about which stage will be the final stage, compliance may be sustained. 
This is why it was expected that inclusion of a termination date in a treaty over freshwater resources changes the compliance pattern of the states.

This expectation was tested by modeling a renegotiation-proof bilateral water allocation agreement between an upstream and a downstream state. After identifying the factors on which compliance is conditioned, the impacts of the knowledge of the termination date on these were studied.

It was found that during the same period of time, culminating at the termination date of the finite agreement, the stability of a treaty with a finite time horizon may be higher, lower or similar to the stability of the same agreement, when it has no pre-known termination date. The relevant factors determining which scenario occurs include: states' perceptions regarding the ability, willingness and capability of the counterpart to impose the different type of sanctions, and the magnitude of such sanctions.

A higher level of stability is expected for finite agreements, if both parties perceive each other as willing and able to use intimidation as a technique to improve their outcome in future negotiations. A finite agreement shows less stability when at least one of the states is seen as permissive or unable to react to the deviation of the other.

An equal level of stability is also possible if both parties are convinced that (1) the other party will not take recourse to any type of sanction in order to change the course of negotiations, and (2) the role of countermeasures in disciplining the free-riding behavior is negligible.

The significance of such findings is that they demonstrate that, in the case of bilateral agreements, setting a termination date for agreements on water allocation is not always a suitable option to enhance resilience to climate change. In fact, there are more imaginable scenarios in which the imposition of a termination date can lead to greater instability. During the negotiations on bilateral treaties for water allocation, negotiators should pay attention to factors such as the power balance between the states and the history of their relations with their counterparts before opting for the usage of agreements with finite time horizons to improve climate change resilience.

Open Access This article is distributed under the terms of the Creative Commons Attribution 4.0 International License (http://creativecommons.org/licenses/by/4.0/), which permits unrestricted use, distribution, and reproduction in any medium, provided you give appropriate credit to the original author(s) and the source, provide a link to the Creative Commons license, and indicate if changes were made.

\section{References}

Ansink, E., Gengenbach, M., \& Weikard, H.-P. (2015). River sharing and water trade. https://papers.ssrn. com/sol3/papers.cfm?abstract_id=2545898. Accessed 06 April 2018.

Ansink, E., \& Ruijs, A. (2008). Climate change and the stability of water allocation agreements. Environmental \& Resource Economics, 41(2), 249-266.

Barrett, S. (1995). The economics of international agreements for the protection of environmental and agricultural resources (Vol. 132). Rome: Food \& Agriculture Org.

Barrett, S. (2003). Environment and statecraft: The strategy of environmental treaty-making. Oxford: OUP.

Barrett, S., \& Stavins, R. (2003). Increasing participation and compliance in international climate change agreements. International Environmental Agreements, 3(4), 349-376.

Bello, J. H. (1996). The WTO dispute settlement understanding: Less is more. American Journal of International Law, 90(3), 416-418.

Bilder, R. (2014). Managing the risks of international agreement. https://papers.ssrn.com/sol3/paper s.cfm?abstract_id=2510910. Accessed 06 April 2018. 
Binmore, K., Rubinstein, A., \& Wolinsky, A. (1986). The Nash bargaining solution in economic modelling. The RAND Journal of Economics, 17(2), 176-188.

Brewster, R. (2009). Unpacking the state's reputation. Harvard International Law Journal, 50, 231.

Cooley, H., Christian-Smith, J., Gleick, P. H., Allen, L., \& Cohen, M. (2009). Understanding and reducing the risks of climate change for transboundary waters (p. 96). Oakland: Pacific Institute.

Cooley, H., \& Gleick, P. H. (2011). Climate-proofing transboundary water agreements. Hydrological Sciences Journal, 56(4), 711-718.

Dinar, S. (2007). International water treaties: Negotiation and cooperation along transboundary rivers. London: Routledge.

Dinar, S., Green, O., McNally, A., Blankespoor, B., \& Kurukulasuriya, P. (2011). Climate change and state grievances: The resiliency of international river treaties to increased water variability.

Eskins, D. (2012). Modeling human decision points in complex systems. Doctoral dissertation, University of Illinois at Urbana-Champaign.

Finus, M. (2003). Stability and design of international environmental agreements: The case of transboundary pollution. International Yearbook of Environmental and Resource Economics, 4(2003), 4.

Goldsmith, J. L., \& Posner, E. A. (1999). A theory of customary international law. The University of Chicago Law Review, 66, 1113-1177.

Goldsmith, J. L., \& Posner, E. A. (2005). The limits of international law. Oxford: Oxford University Press.

Guzman, A. T. (2008). How international law works: A rational choice theory. Oxford: Oxford University Press.

Hamner, J. H., \& Wolf, A. T. (1998). Patterns in international water resource treaties: The transboundary freshwater dispute database. Colorado Journal of International Environmental Law and Policy, 9, 157.

Harstad, B. (2008). How to negotiate and update climate agreements. Discussion Paper 08-19, Harvard project on international climate agreements, Belfer Center for Science and International Affairs, Harvard Kennedy School, November.

Hathaway, O. A. (2005). Between power and principle: An integrated theory of international law. The University of Chicago Law Review, 72, 469-536.

Heister, J. (1997). Der internationale CO2-Vertrag: Strategien zur Stabilisierung multilateraler Kooperation zwischen souveränen Staaten (Vol. 282). Tübingen: JCB Mohr (Paul Siebeck).

Hovi, J., \& Areklett, I. (2004). Enforcing the climate regime: Game theory and the Marrakesh accords. International Environmental Agreements, 4(1), 1-26.

Intergovernmental Panel on Climate Change. (2014). Climate change 2014-impacts, adaptation and vulnerability: Regional aspects. Cambridge: Cambridge University Press.

International Law Commission. (2001). Draft articles on responsibility of states for internationally wrongful acts. Yearbook of the International Law Commission, 2(2), 49.

Koremenos, B. (2001). Loosening the ties that bind: A learning model of agreement flexibility. International Organization, 55(2), 289-325.

McCaffrey, S. C. (2003) The need for flexibility in freshwater treaty regimes. In Natural resources forum, 2003 (Vol. 27, pp. 156-162). Wiley Online Library.

McEvoy, D. M., \& Stranlund, J. (2006). Enforcing'Self-Enforcing'International Environmental Agreements.

Neumayer, E. (2017). Greening trade and investment: Environmental protection without protectionism. London: Routledge.

Rinceanu, J. (2000). Enforcement mechanisms in international environmental law: Quo vadunt. Journal of Environmental Law and Litigation, 15, 147.

Sand, P. H. (1997). Commodity or taboo? International regulation of trade in endangered species. In H. O. Bergesen \& G. Parmann (Eds.), Green globe yearbook of international co-operation on environment and development (pp. 19-36). Oxford University Press.

Stinnett, D. M., \& Tir, J. (2009). The institutionalization of river treaties. International Negotiation, 14(2), 229-251.

United Nations. (1969). Vienna Convention on the law of treaties, 23 May 1969. UN.

United Nations. (1992). Convention on the protection and use of transboundary watercourses and international lakes: Done at Helsinki, on 17 March 1992. UN.

United Nations. (1997). Convention on the law of non-navigational uses of international watercourses, on 21 May 1997. UN.

Wilby, R. L., \& Dessai, S. (2010). Robust adaptation to climate change. Weather, 65(7), 180-185.

Wolf, A. T. (2002). Atlas of international freshwater agreements (Vol. 4). UNEP/Earthprint.

Yoffe, S., Wolf, A. T., \& Giordano, M. (2003). Conflict and cooperation over international freshwater resources: Indicators of basins at RISR. JAWRA Journal of the American Water Resources Association, 39(5), 1109-1126. 